\title{
EXTRA-DURAL ANALGESIA IN OBSTETRICS AND GYNÄECOLOGY
}

\author{
By C. J. Massey Dawkins, M.D., D.A., F.F.A.R.C.S. \\ Senior Anaesthetist, Uuiversity College Hospital
}

There are many advantages to be derived from the use of extra-dural block in obstetrics and gynaecology. In the past many anaesthetists have been reluctant to make use of the method owing to the difficulty in accurately locating the extra-dural space, but with advances in technique this has been overcome and there has been a great increase in the use of this form of analgesia in recent years. One of the great advantages in maternity work is that there is a minimal upset of metabolism and the drug does not pass the placental barrier so that the foetus is quite unaffected. While in operative gynaecology where the patient must be in the Trendelenburg position, the weight of the bowels on the unparalysed diaphragm does not interfere with the respiratory exchange, and consequently bleeding is much reduced. The technique must be considered under four headings, operative gynaecology, therapeutic relief of such conditions as phlegmasia alba dolens, Caesarean section and normal labour.

\section{Anatomy}

The extra-dural space (also called epidural or peridural) is formed by the splitting into two layers of the dura mater when it emerges from the foramen magnum. The outer or parietal layer forms the periosteal lining of the vertebral canal. The inner or parietal layer surrounds the spinal cord. The space is closed below by the posterior sacro-coccygeal ligaments at the sacral hiatus and it is pierced laterally by openings for the $3 I$ pairs of spinal nerves as they emerge from the intervertebral foramina. The space has an average width of 3-4 $\mathrm{mm}$. and is loosely filled with fatty connective tissue and a plexus of veins and arteries. Injection of radio-opaque substances into the space shows a rapid spread into the paravertebral space through the intervertebral foramina, and it is in this region that local analgesics injected into the space have their effect, as there is very little absorption through the dura mater. The fibres of the mixed spinal nerves and the grey and white rami communicantes of the sympathetic system will all be affected by the block, though the effect on 画e motor nerves will be less than the others, owi to the larger size of the fibres and the presence ofva tougher sheath. Fluid injected into the extra-duragl space cannot reach the brain owing to the fusion of the two layers of dura mater at the foramen magnum.

\section{Physiology}

In the upright position the extra-dural spare remains only a potential one as the two layers of dura mater are in contact. When the spine 3 s flexed, separation of the layers occurs with formation of a temporary negative pressure garying between $-\mathrm{I}$ and $-18 \mathrm{~mm}$. of mercury. प9ks negative pressure is only present in $84 \%$ of pateres and the reason for this is unknown. But greatuse can be made of it in the location of the space, by attaching a small capillary manometer to the neefle used for injection. Assuming that the patient hagla negative pressure this will at once be apparent $\overline{\text { gn }}$ the manometer when the space is tapped. In those patients who do not possess a negative pressuge, the space can be found by the sign of loss of resistance to the advancing needle as it emergess from the ligamentum flavum. The identification of the extra-dural space depends on the utilization of one or other of these two methods, and these require explanation in greater detail.

\section{A. Recognition of Space by Presence of Negatise Pressure.}

If a small drop of saline is placed in the hub $\frac{{ }^{\circ}}{\text { of }}$ the needle, it will be sucked into the shaft of the needle when the space is tapped. This was called the 'signa da gota' and it was modified by Odoum who obtained a much more accurate result by substituting a small piece of capillary glass tubing containing a bubble of air in saline. This type of indicator is extremely sensitive, especially if the saline is coloured by a dye such as $\mathrm{I} \%$ eosin. $\mathbb{T}_{\mathrm{n}}$ order to obtain an air-tight joint between the needle and the capillary tube, it is essential to use a needle with a large hub such as the Wilfrgd 
Harris, so that no artefact caused by loosening the joint could be brought about by gripping the hub of the needle by the finger and thumb. It is preferable to use a fairly stout needle such as I 8 SWG and to shorten it to $6 \mathrm{~cm}$. In the $16 \%$ of patients who do not exhibit a negative pressure, the feel of loss of resistance of the advancing needle indicates that the space has been tapped and this can be confirmed by means of the air test described in the next paragraph.

\section{B. Recognition of Space by Sign of Loss of Resistance.}

When the bevel of the needle leaves the ligamentum flavum and enters the epidural space, this fact is at once apparent as resistance ceases. If a small glass syringe containing $2 \mathrm{cc}$. of air or saline be attached to the hub of the needle and the thumb kept on the end of the piston, then the moment the space is tapped the piston will be felt to slide forward without resistance. Various mechanical devices have been produced to accentuate this result, such as Macintosh's spring loaded trocar inside the needle and the Ilké spring loaded syringe. The simplest mechanical device is the author's elastic band with a hole for the nozzle of the syringe, which is slipped round the whole syringe. Any device making use of a syringe attached to the needle is, however, clumsy in use and suffers from the inherent disadvantage that the same sense of loss of resistance occurs when the needle has gone too far and the dura mater has been pierced.

Whichever method of identification of the epidural space is adopted, it should always be adhered to in order that constant repetition may increase the dexterity of the operator. Similarly needles of the same size and bore should always be used in order that slight variations in pressure may be instantly recognized.

If by accident the dura mater is pierced and cerebrospinal fluid drips back from the hub of the needle, then the needle should be reinserted in the adjacent space above or below. Very occasionally a blood vessel is injured and blood appears at the hub. Reinsertion as before is advisable.

\section{Dosage and Site of Injection}

For operations such as hysterectomy where it is necessary to block spinal nerves T8-TI2, the injection should be made between the spines of $T_{12}$ - LI with the patient in a $20^{\circ}$ head down tilt. This tilt will cause the solution to run up the epidural space to block the required nerves. A total volume of $22-24$ cc. should be used, but if the patient is very fat, the epidural space owing to the amount of fat it possesses, will be correspondingly larger and it will be advisable to increase the dose by $3 \mathrm{cc}$. A deduction of the same amount may be made for $a \stackrel{2}{2}$ very thin patient.

For operations on the perineum, the injection $\stackrel{\varrho}{c}$ should be made between $\mathrm{L}_{4}-\mathrm{L}_{5}$ and as soon as the space is located the patient should be tilted $20^{\circ} \stackrel{\overrightarrow{5}}{\stackrel{5}{+}}$ head up and an injection of 16 - $18 \mathrm{cc}$. made with $\overline{0}$ variations as above. The head up position should 들 be maintained for 6 minutes but it is advisable to $\frac{\bar{D}}{\bar{D}}$ put the legs into the lithotomy position at the same $\underset{\mathbb{Q}}{\stackrel{0}{2}}$ time, as no patient who has a potentially unstable vaso-motor system should ever be in a position where the head is higher than the legs, to prevent $\vec{\circ}$ possible cerebral anoxia.

The analgesic drug of choice is Xylocaine in a strength of $1 . \frac{3}{4} \%$ together with adrenaline $\bar{C}$ $1 / 250,000$. This concentration will be effective for 3 . about 2 hours or a little longer if the patient receives nitrous oxide and oxygen throughout. Analgesia $\stackrel{N}{\omega}$ will come on between 6 and 9 minutes from the $\omega$ injection. If it is desired that the patient remain or awake during the operation, then it must be ex- $\vec{\perp}$ plained that the sense of touch is not completely 음 abolished and that a sensation of something pressing on the abdomen will be experienced. $z$ Most patients however desire to be unconscious and the drug of choice is soluble hexobarbitone $\frac{\mathbb{}}{3}$ (Evipan) $\frac{1}{2}-I \mathrm{~g}$. This is to be preferred to thiopentone as it lasts longer, does not cause laryrof geal spasm and does not depress the respirato centre. If the operation is to be a major procedue such as the Howkins synchronous combined pelvi excentration, then an indwelling nylon catheter should be passed into the space via a Tuohy needle and repeated injections of $6-8 \mathrm{cc}$. of solution made when necessary. The catheter may with advantage $\frac{\otimes}{\mathbb{Q}}$ be left in at the close of the operation and analgesia $\stackrel{\Omega}{\rightleftarrows}$ continued throughout the post-operative period, no pain relieving drugs then being necessary.

In operations on the lower abdomen, a certain number of sympathetic ganglia will be included in the block and there will be in consequence, some $\frac{}{3}$. fall of blood pressure, depending on the height to which the block reaches. This fall may be pre- 3 . vented by giving a pressor drug such as ephedrine as soon as the block is completed. An average dose would be $\frac{3}{4} \mathrm{gr}$., of which $\frac{1}{4}$ gr. is given intravenously and the remainder intramuscularly. It will occasionally be necessary to repeat this but a major $\frac{D}{0}$ fall of pressure, as might occur with a spinal, is most unlikely. In perineal operations it may be $\mathcal{N}$ desirable to operate with a bloodless field, and in N these cases the pressor drug is omitted. Experi- N ments have shown that the blood loss from a $\frac{\omega}{\sigma}$ vaginal hysterectomy done under an epidural block, is exactly half that of a similar operation done with $\stackrel{\varrho}{C}$ a muscle relaxant plus general anaesthesia. It is important to remember that the blood pressure? should be restored to within $80 \%$ of normal before 
all the stitching up in a colporrhaphy is done, lest reactionary haemorrhage takes place. Neglect of this precaution will bring the avascular technique into disrepute.

\section{Technique}

It is important that the patient receive adequate premedication. A suitable combination of drugs is Nembutal $\mathrm{I} \frac{1}{2} \mathrm{gr}$. by mouth and Omnopon $\mathrm{I} / 3 \mathrm{gr}$. and Scopolamine I/I 50 gr. by injection one hour before operation. This will, in the majority of cases, ensure a retrograde amnesia. The patient is placed in the lateral position as for a spinal. All equipment must be autoclaved and gloves must be worn. A skin wheal with Xylocaine over the chosen interspace is performed and then the $6 \mathrm{~cm}$. needle is introduced into the extra-dural space. Failure to locate the space owing to bony obstruction indicates insufficient flexion of the spine. If owing to age of the patient and ossification of the ligaments it is impossible to check in time the onward course of the needle and the theca is entered, then injection should be made in an adjacent space. Repeated dural puncture must result in abandonment of the method.

Assuming that with the devices already described the space is satisfactorily located, then a test injection of $4 \mathrm{cc}$. of the solution is made and after a pause of 4 minutes the patient is asked to move her legs. Failure to do this would indicate an accidental spinal and the operation should be performed under this. If motor power is unaffected then the remainder of the solution is given.

During the injection of the major part of the solution it will invariably be found that the patient will commence to breathe deeply. This is due to a temporary compression of the cerebro-spinal fluid by the injected solution. This causes a rise in intracranial pressure and a stimulus to the respiratory centre. This is a valuable confirmatory sign that the solution is in the right place. The patient should be warned of transient giddiness.

If any of the injected solution drips back when the syringe is detached from the needle, the anaesthetist may perhaps wonder if this is cerebro-spinal fluid. If the wrist is ungloved and a drop allowed to fall on it, then all is well if the fluid is at room temperature. The effect may be enhanced by standing the solution in an ice pack before use. If when the syringe is removed a drip back containing a cluster of tiny bubbles is observed, this is a further absolutely diagnostic sign that the needle is in the space. The reason for this is unknown.

When confidence has been gained, the safety pause may be omitted and the patient should be anaesthetized before the block. In the author's experience the safety pause is quite unnecessary and may be distressing to the patient.
As soon as the block is completed the patient is turned on to her back and a blood pressure cuff fitted on the arm and a reading taken before and after the injection of the pressor drug if this is given. The patient is ready for surgery after $6-9$ minutes. It is important to remember that the vagus nerve is not included in the block and if when the peritoneum is open the surgeon explores the abdomen and tugs on any viscera, then the patient may retch or wake up. This may be avoided by giving a small supplementary injection of Evipan as the peritoneum is incised. As the patient is so lightly asleep throughout, it is essential to see that the arms are properly strapped to the table. At the conclusion of the operation, the patient may be woken up by sponging the face with cold water and encouraged to do breathing exercises. The remarkable freedom from post-operative chest complications encountered with this technique is largely due to this ability of the patients to breathe without pain.

The patient must be transported back to bed with the feet higher than the head; not because of any fall of blood pressure but in order to make sure that the nursing staff never have her in the head up position until she is in bed. The foot of the bed should be blocked until the blood pressure has remained over $100 \mathrm{~mm}$. of mercury for over 2 hours Subject to the orders of the surgeon the patient should be encouraged to eat solid food as soon as possible. This helps to reduce the incidence of post-operative distension.

\section{Complications}

In about $\mathbf{I} \%$ of cases bony obstruction or excess fat makes the location of the space impossible. In a further $1 \frac{1}{2} \%$ the analgesia will be imperfect and a supplementary general anaesthetic will be required. This is probably due to the failure of the solution to reach every nerve segment even though it is properly placed and it is caused by an abnormal attachment of the ligaments surrounding the intervertebral foramen to the mixed spinal nerve, so that the solution does not emerge from that particular foramen, and analgesia is absent.

If despite all precautions an accidental spinal is given, no harm will come to the patient provided that artificial respiration is performed and a careful watch kept on the blood pressure. The average duration of paralysis of the body with $1 \frac{3}{4} \%$ Xylocaine is 75 minutes and respiration will be adequate within 5 minutes from the restart. It is not even necessary to inform the surgeon of the mishap. This freedom from anxiety is peculiar to Xylocaine and in many recorded cases there has been no fall of blood pressure despite dilated pupils and a paralysed diaphragm. The rationale of this would appear to be impossible of explanation. 
In about $\mathrm{I} \%$ of cases there will be a fall of blood pressure of more than $50 \%$ and it may be necessary to put up an adrenaline drip. This should be of a concentration of $\mathrm{I} / 250,000$. Most cases however respond to repeated small amounts of a pressor drug. The fall of pressure is never so great or so long lasting as that occurring with spinal analgesia.

The advantages of extra-dural block compared with other forms of anaesthesia are summarized in the accompanying table.

\section{Therapeutic Indications}

The paralysis of the sympathetic ganglia associated with extra-dural block may be turned to advantage in any pathological condition where the blood supply is impaired. The outstanding example in obstetrics is phlegmasia alba dolens due to venous thrombosis. A block of the sympathetic nerve supply to the leg will cause a vaso-dilatation of the collateral circulation in the veins and the swelling will speedily subside. The block may consist either of repeated injections once daily of $20 \mathrm{cc}$. of $\mathrm{I} \%$ Xylocaine between $L_{2}-L_{3}$, the patient lying on the affected side, or an indwelling catheter may be used.

Toxaemias of pregnancy which cause anuria can be quickly relieved by interruption of the sympathetic vaso-constrictor impulses to the kidney. This causes an increased blood flow and so restores the secretory function. In cases of eclampsia advantage may be taken of the associated sensory analgesia to perform Caesarean section if this is indicated.

In cases of severe dysmenorrhoea which have not responded to symptomatic treatment, it may in the last resort be necessary to perform a lumbar sympathectomy. The success of this operation can be anticipated if a preliminary extra-dural block is introduced when pain is at its height. Temporary relief of pain will show that permanent section of the nerves will bring lasting relief.

\section{Caesarean Section}

The indications for this operation, from the point of view of the anaesthetist, fall into two main classes. Firstly, the elective section, performed on a predetermined date, for pelvic disproportion or for a breach presentation in an elderly primipara. So long as the anaesthetist realizes that he has two patients to deal with instead of one, the choice of anaesthetic is immaterial. Although it must be remembered that the foetal death rate is still, despite every care, about twice as great as that of normal labour.

Secondly, emergency section, for placenta praevia, obstructed labour or toxaemia. In these cases it is of paramount importance that the operation should be conducted under the method of anaesthe- sia which causes the least possible disturbance of an already impaired metabolism. Extra dural block is, in the opinion of the author, the method of choice in these cases. As the drug does not pass the placental barrier, provided that anoxia in the mother is avoided, the foetus is completely unaffected by the block. And the upset to the metabolism of the mother is minimal.

The technique of the block does not differ from that for a hysterectomy but it must be remembered that the blood pressure in the pregnant woman is very labile and it is advisable to give the pressor drug before the block is put in. It is preferable to have the patient conscious throughout and if this is objected to, stress should be laid on the fact that the life of the foetus is endangered by general anaesthesia. The retention of the sense of touch must be explained to the mother. It may help her to have a stream of oxygen playing on her face throughout the operation. The baby invariably cries as soon as it is delivered from the wound and it is of considerable advantage to show the baby to the mother at this point, as the maternal sentiment will have a markedly beneficial effect on the contraction of the uterus. The mother should be returned to bed with a slight head down tilt and should be encouraged to resume normal feeding habits as soon as possible as this results in a considerable reduction of post-operative distension which is such a common complication of this operation. The nursing staff show a marked preference for this method of anaesthesia as the postoperative condition of the patient is much superior compared with other forms of anaesthesia.

\section{Normal Labour}

The use of caudal analgesia to relieve the pains of labour has long been known, but the necessity of repeated injections with consequent increased risk of sepsis has prevented the widespread use of the method. In 1942, Hingson and Edwards published a paper on the use of continuous caudal block using an indwelling needle. They found that labour could be made entirely painless, without inhibiting uterine contraction and with no harmful effect on the foetus. The motor nerve supply of the upper uterine segment is derived from the thoracic sympathetic ganglia down to Tro, while the sensory nerve supply comes from TiI and Ti2. The motor and sensory nerve supply of the lower uterine segment, cervix and vagina is derived from the parasympathetic system via $\mathrm{S}_{2}$, $\mathrm{S}_{3}$ and $\mathrm{S}_{4}$. Provided that an analgesic solution does not reach higher than TIo in the epidural space, labour will be painless and uterine contractions unaffected. Reports covering over 2,000,000 cases have now been published and as the accompanying table shows drawbacks are very few. 
Epidural ANalgesia compared with other Forms of ANaEsthesia

\begin{tabular}{|c|c|c|}
\hline $\begin{array}{l}\text { Advantages over } \\
\text { intercostal block }\end{array}$ & $\begin{array}{l}\text { Advantages over } \\
\text { spinal analgesia }\end{array}$ & $\begin{array}{l}\text { Advantages over } \\
\text { general anaesthesia } \\
\text { plus curare }\end{array}$ \\
\hline
\end{tabular}

(Modern Practice in Anaesthesia-Frankis Evans)

Advantages of Continuous Caudal Analgesia

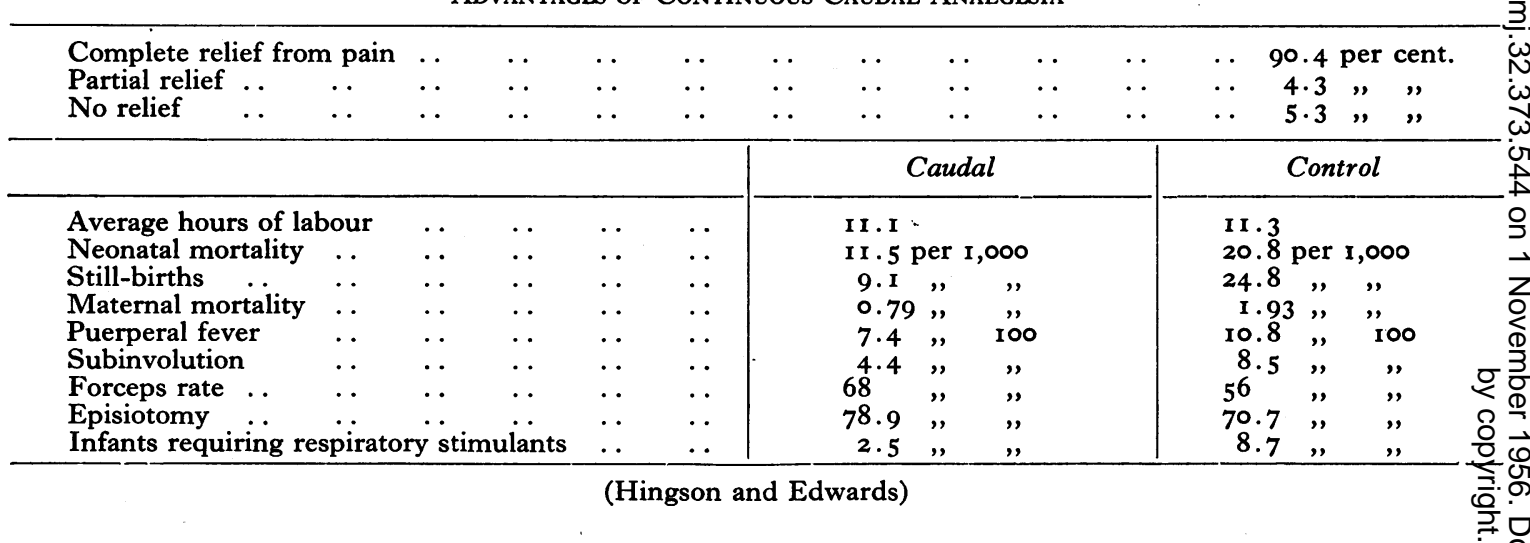

Obstetricians in this country have been slow to take up the method largely because of the $80 \%$ forceps rate and the need for the continuous attendance of a trained anaesthetist, but in a maternity unit one anaesthetist could easily look after several cases in labour at the same time.

\section{Anatomy}

The sacrum is formed by the fusion of the five sacral vertebrae but owing to the variation in the fusion of the arches of these vertebrae a normal sacrum can hardly be said to exist. The caudal space, which is that part of the epidural space lying below the fifth lumbar vertebra, is bounded in front by the body of the sacrum and behind by the arches of the vertebrae. The posterior boundary is called the sacral hiatus and this is closed by the posterior sacrococcygeal ligaments. The hiatus is triangular in shape and in $34 \%$ of patients its apex is formed by the fusion of the arches of the fourth sacral vertebra, while on either side there lie the sacral cornua which represent the articular surfaces of the fifth sacral vertebra. These cornua are easily felt through the skin and constitute landmarks to the hiatus. In $19 \%$ the hiatus is nearer the coccyx due to the fusion of the arches of the fifth sacral vertebra and is correspondingly much smaller in size. In nearly $47 \%$ the hiatus is dis-⿳亠二口犬 placed upwards owing to the non-fusion of the third, second or first sacral vertebrae and in $0.3 \% \stackrel{\mathbb{D}}{\circ}$ there is no posterior bony wall at all.

The dura mater normally ends at the lower bor-윽 der of second sacral vertebra and the average distance between it and the hiatus is $47 \mathrm{~mm}$., but: extremes of $19 \mathrm{~mm}$. and $75 \mathrm{~mm}$. have been noted. The volume of the sacral canal varies between 123 and $65 \mathrm{cc}$. In many sacra there are bands of 0 fibrous tissue passing from the anterior to the 3 . posterior wall and these have the effect of splitting up the space into loculi which are undoubtedly the cause of the partial analgesia which is sometimeso obtained, injection having been made into one loculus.

\section{Technique}

Three things are necessary for a successfuln caudal block : I. The needle must pierce the sacral hiatus and
enter the caudal space.

2. The dura mater must be avoided.

3. The analgesic solution must reach the re- $\mathscr{\Phi}$ quired level. 
With the patient in the left lateral position, a skin wheal with any local analgesic is made over the sacral hiatus, but it should be kept as small as possible in order not to obscure the landmarks of the sacral cornua. A 16 or 17 SWG needle is then passed through the wheal at right angles to the skin until it is felt to impinge on the anterior wall of the sacral canal. It is then slightly withdrawn and reinserted parallel to the plane of the body of the sacrum and advanced for a distance of $15 \mathrm{~mm}$. A distinct snap is sometimes felt as it traverses the sacro-coccugeal ligament. A nylon catheter is then passed through the needle and the needle is withdrawn over it. The catheter is connected to a syringe or a reservoir of analgesic solution and an injection of $8 \mathrm{cc}$. is made into the caudal canal. The customary pause to rule out accidental spinal block is then made. After five minutes wait the patient is asked to move her legs. If motor power is unaffected then a further injection of $22 \mathrm{cc}$. is made and within 20 minutes labour will become pain-free. The analgesic of choice is $1 \frac{1}{2} \%$ Metycaine and this is put up in sterile flasks containing $100 \mathrm{cc}$. to which the catheter is connected by means of a syringe and a three way tap. The whole outfit with the exception of the flask can be autoclaved and connection is made to the flask by peeling off a protective cap and plunging the connecting needle through this. Further injections of $20 \mathrm{cc}$. are made every $30-40$ minutes. Provided that the solution does not reach higher than the tenth thoracic vertebra labour will be painless and uterine contractions unaffected and there will be no harmful effect on the foetus whatever.

\section{Effects}

The course of labour will be altered by a length- ening of the second stage as the normal impulse to push out the foetal head will not be appreciated by the analgesic patient and in a primipara it will be necessary to extract the baby with forceps, but with the relaxed perineum forceps extraction is considerably easier than it would otherwise be, and if episiotomy is necessary it will be painless. In a multipara who has had a previous delivery under light general anaesthesia, forceps are rarely necessary as the mother knows what to do when she is told to bear down. The advantages of the method are summarized in the accompanying table.

\section{Disadvantages}

The somewhat complicated technique requires the services of a trained anaesthetist, and failure to achieve analgesia cannot be ruled out owing to possible abnormalities of the sacrum or even inability to locate the hiatus owing to obesity of the patient. Repeated blood pressure readings are necessary and a hand must be kept on the fundus of the uterus in order to recognise the onset of a contraction. Gross anaemia, low blood pressure, skin sepsis over the sacrum and a history of rapid delivery at a previous labour are contraindications. Errors in technique include accidental puncture of the dura mater, insertion of the needle posterior to the sacrum (which can be recognized by keeping a finger over the sacrum during the injection and feeling the occurrence of surgical emphysema) and the injection of too great a quantity of solution with resultant paralysis of the motor nerve supply of the uterus followed by uterine inertia. The safest form of treatment of this complication for mother and child is an immediate Caesarean section.
H. K. LEWIS \& Co. Ltd. Medical Publishers and Booksellers

136 GOWER STREET LONDON, W.C. 1 (Adjoining University College and Hospital) Telephone: EUSton $\mathbf{2 8 2}$ (7 lines)

Telegroms: Publicavit, Westcent, London Estoblished 1844

\section{Medical Lending Library}

ANNUAL SUBSCRIPTION from $\mathrm{EI}$ 17s. 6d. Prospectus post free on application

Bi-monthly List of New Books and New Editions added to the Library sent post free on request

The Library Catalogue revised to December, 1949, containing a classified index of authors and subjects.

To subscribers $17 / 6$ net; To non-subscribers 35/- net. Postage 1/6 Supplement 1950 to 1952 . To subscribers $1 / 6$ net; to nonsubscribers $3 /-$ net; postage $6 \mathrm{~d}$.

NEW BOOKS ADDED IMMEDIATELY UPON PUBLICATION 